\title{
Lignocellulose structure and the effect on nanocellulose production
}

\begin{abstract}
Lignocellulose as a biopolymer material consists of three constituents, including hemicellulose, lignin, and cellulose. Nanocellulose as nanodimension cellulose is one of the components of lignocellulose, which is a biopolymer material. Through the depolymerization of lignocellulosic biomass, cellulose is extracted with excellent properties for many applications. The biomass with different source, type and species has different structures and then different properties, such as strength of lignin layer, crystallization of cellulosic area and accessibility to chemicals. Therefore, biorefinery processes to extract cellulose from lignocellulose biomass should be developed in order to deconstruct noncellulosic materials in lignocellulose while maintaining cellulose products for further hydrolysis into nanocellulose material. In this chapter, the effects of lignocellulose structure and composition on the production of nanocellulose is discussed.
\end{abstract}

Keywords: Cellulose; Depolymerizing; Lignocellulosic biomass; Nanocellulose; Noncellulosic material; Polymer 